\title{
Diffuse Idiopathic Skeletal Hyperostosis (DISH) and non small cell lung cancer: case presentation and review of the literature
}

The authors declare no financial disclosure

\begin{abstract}
Diffuse idiopathic skeletal hyperostosis (DISH), also known as Forestier's disease, is a systemic non inflammatory disease of unknown cause. It is characterized by the presence of osteophytes due to calcification and ossification of spinal ligaments and entheses. Moreover, diffuse idiopathic skeletal hyperostosis has been associated with a variety of metabolic disorders. However, to the best of our knowledge no association with non small cell lung cancer (NSCLC) has been reported so far. In the present study we report a case of a patient with NSCLC and DISH.
\end{abstract}

Key words: DISH, lung cancer, bronchoscopy

Pneumonol Alergol Pol 2016; 84: 116-118

\section{Introduction}

Diffuse idiopathic skeletal hyperostosis (DISH) is a non inflammatory disease of unknown cause, characterized by ossification of spinal ligaments and entheses [1]. Although it has been associated with a variety of disorders, no association with non small cell lung cancer (NSCLC) has been reported so far, to the best of our knowledge. In the present study we report a case of a patient with NSCLC and DISH.

\section{Description of case}

A 65 year-old Caucasian male smoker (60 pack years) was admitted to our department complaining for minor dyspnea on exertion. He did not report any other symptom and his medical history was unremarkable.

Physical examination revealed digital clubbing and hypertension that did not need persistent treatment. Blood analysis was normal. Chest X-ray
(Figure 1) showed a tumor-like opacity in the left upper lobe and calcification along the right anterolateral aspects of the vertebral bodies of

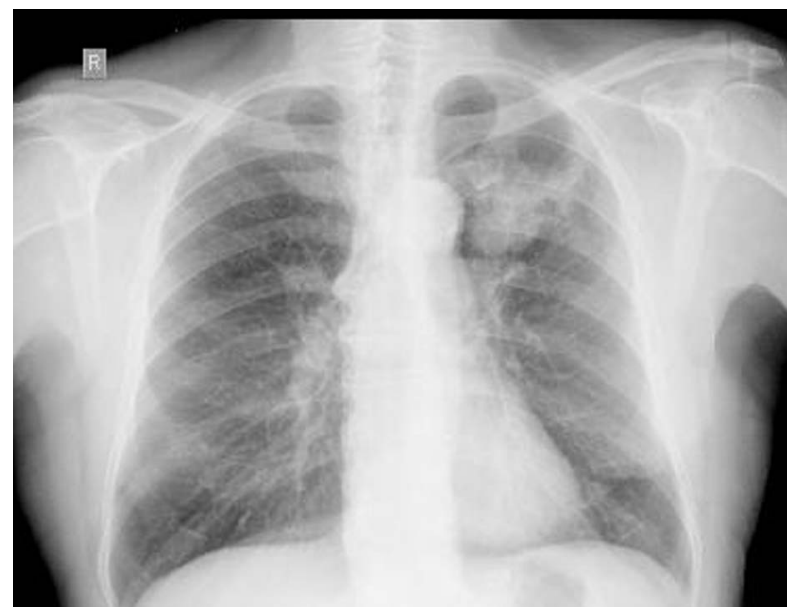

Figure 1. Chest $\mathrm{X}$-ray revealed a nodular opacity in the left upper lobe and calcification along the right anterolateral aspects of the vertebral bodies of the thoracic spine

Address for correspondence: loannis Tomos, $2^{\text {nd }}$ Pulmonary Medicine Department, "Attikon" University Hospital, 1, Rimini Street, 12462 Haidari, Athens, Greece.

Tel: +302105831184, Mobile; +306942707287, e-mail address: etomos@hotmail.com

DOI: 10.5603/PiAP.2016.0010

Received: 20.11.2015

Copyright (C) 2016 PTChP

ISSN 0867-7077 


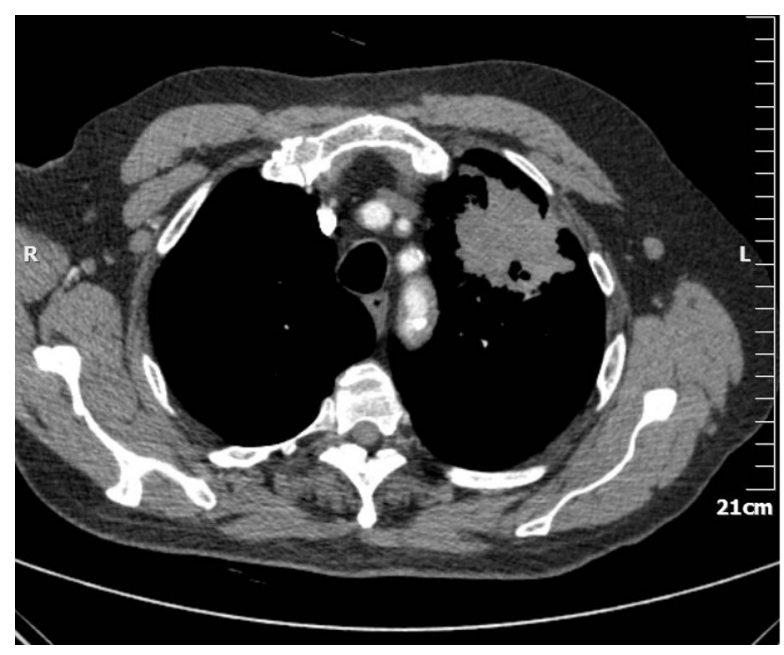

Figure 2. Chest Computerized Tomography (CT) confirmed the presence of a pulmonary mass in the left upper lobe

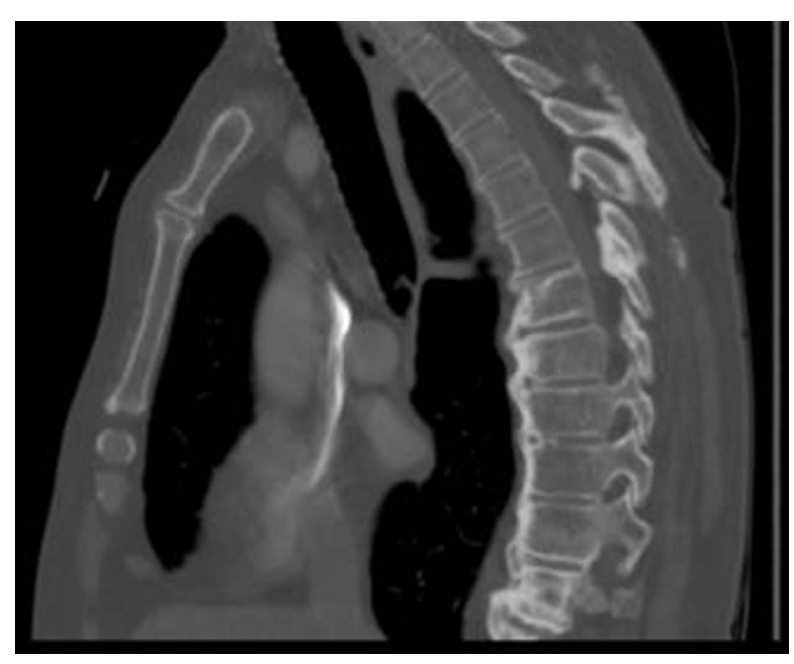

Figure 3. Calcification and osteophytes along the right anterolateral aspect of four contiguous thoracic vertebral bodies

the thoracic spine. Chest computed tomography (CT) confirmed the presence of a pulmonary mass in the left upper lobe as well as calcification and osteophytes along the right anterolateral aspect of four contiguous thoracic vertebral bodies (Figs 2, 3). The intervertebral height in the involved segment was well preserved without any degenerative disc disease and without any involvement of the sacroiliac joint. Bronchoscopy had no abnormal findings. The patient was submitted to fine needle biopsy of the lung lesion under CT guidance. The histological examination revealed adenocarcinoma of the lung.

\section{Discussion}

We present a case of a patient with NSCLC and diffuse idiopathic skeletal hyperostosis. The diagnosis of DISH is based mainly on radiological features. Resnick's criteria are more often used and require at least four contiguous vertebrae to be involved, preservation of the intervertebral disc space and absence of any apophyseal joints or sacroiliac participation [2]. The case we present fulfills all these criteria. Usually, DISH concerns the anterolateral aspect of the vertebrae, what is compatible with the linear paravertebral opacity we observed on chest imaging [3]. The cause of DISH remains uncertain [4]. However, a variety of factors have been proposed to contribute to its pathogenesis, including mechanical (location of the aorta), environmental, related to treatment (derivatives of vitamine A) as well as genetic predisposition (HLA polymorphisms). Increased levels of growth hormone and insulin-like growth factor-1(IGF-1) have been observed in patients with DISH [5-7]. Moreover, a higher prevalence of DISH has been reported in patients with metabolic disorders, such as obesity, hypertension, diabetes mellitus, hyperuricemia, dyslipidemia and hyperinsulinemia [6-8]. Our patient, did not suffer from any of the above mentioned comorbidities except hypertension that however, did not need persistent treatment.

Usually, patients are asymptomatic, exactly just as our case and the diagnosis of DISH is based on an incidental finding in chest imaging. However, patients may present local symptoms depending on the distribution of the osteophytes, such as neck, thoracic spine or lumbar pain. Dysphagia or even airway obstruction have also been described due to osteophytes in the cervical spine [9, 10].

In the differential diagnosis a variety of diseases that appear with similar characteristics with DISH, such as osteoarthritis of the vertebral column and ankylosing spondylitis should be considered. In our case, the absence of degeneration of intervertebral disks as well as of any erosion and bone ankylosis of the sacroiliac joints excludes the above diseases.

On the other hand, lung cancer consists of the most common cause of cancer-related mortality worldwide [11]. Up to $85 \%$ of all cases concern NSCLC, while adenocarcinoma accounts for more than $50 \%$ of the cases [12]. So far, DISH is considered to be idiopathic and its etiology remains unclear. However recently, there is evidence that in lung cancer patients and especially NSCLC, IGF-1 plasma levels are increased [13], whilst several studies have shown the potential role of IGF-1 in DISH pathogenesis by increasing osteoblastic activity [5, 14]. The mentioned role of IGF-1 in the pathogenesis of DISH and its increased levels 
found in NSCLC patients, potentially, may imply an association between the diseases. Further studies are needed to confirm the above hypothesis. Unfortunately, concerning our patient, it was not feasible to measure his IGF-1 plasma levels.

\section{Conclusion}

We present a rare case of a patient with concurrent NSCLC and DISH, a disease with no special symptoms and thereafter usually underdiagnosed. Although DISH is considered idiopathic, the increased levels of IGF-1 in NSCLC and its role in osteoblastic activity and the pathogenesis of DISH, give a hint for a potential association between the diseases. However, this coexistence of DISH and NSCLC might be just incidental and not necessary clinically significant. Unfortunately, so far, there is not enough evidence to confirm any clinical associations between both disorders, while further research is needed to elucidate the link that might exist.

\section{Conflict of interest}

The authors declare no conflict of interest.

\section{References:}

1. Utsinger PD. Diffuse idiopathic skeletal hyperostosis. Clin Rheum Dis 1985; 11: 325-351.

2. Resnick D, Niwayama G. Diffuse idiopathic skeletal hyperostosis (DISH): ankylosing hyperostosis of Forestier and Rotes-Querol. Diagnosis of Bone and Joint Disorders. $2^{\text {nd }}$ ed. Philadelphia, PA: WB Saunders 1988: 1563-1615.
3. Resnick D, Niwayama G. Radiographic and pathologic features of spinal involvement in diffuse idiopathic skeletal hyperostosis (DISH). Radiology 1976; 119: 559-568.

4. Mader R. Current therapeutic options in the management of diffuse idiopathic skeletal hyperostosis. Expert Opin Pharmacother 2005; 6: 1313-1318.

5. Denko CW, Boja B, Malemud CJ. Growth hormone and insulin-like growth factor-I in symptomatic and asymptomatic patients with diffuse idiopathic skeletal hyperostosis (DISH). Front Biosci 2002; 7: a37-43.

6. Sarzi-Puttini P, Atzeni F. New developments in our understanding of DISH (diffuse idiopathic skeletal hyperostosis). Curr Opin Rheumatol 2004; 16: 287-292.

7. Sencan D, Elden H, Nacitarhan V, Sencan M, Kaptanoglu E. The prevalence of diffuse idiopathic skeletal hyperostosis in patients with diabetes mellitus. Rheumatol Int. 2005; 25: 518-521.

8. Nascimento FA, Gatto LA, Lages RO, Neto HM, Demartini Z, Koppe GL. Diffuse idiopathic skeletal hyperostosis: A review. Surg Neurol Int 2014; 16; 5: S122-5. doi: 10.4103/21527806.130675 .

9. Hwang JS, Chough CK, Joo WI. Giant anterior cervical osteophyte leading to Dysphagia. Korean J Spine. 2013; 10: 200-202. doi: 10.14245/kjs.2013.10.3.200.

10. Lin HW, Quesnel AM, Holman AS, Curry WT Jr, Rho MB. Hypertrophic anterior cervical osteophytes causing dysphagia and airway obstruction. Ann Otol Rhinol Laryngol 2009; 118: $703-707$.

11. Mirsadraee S, Oswal D, Alizadeh Y, Caulo A, van Beek E Jr. The $7^{\text {th }}$ lung cancer TNM classification and staging system: Review of the changes and implications. World J Radiol 2012; 4: 128-134. doi: 10.4329/wjr.v4.i4.128.

12. Zugazagoitia J, Enguita AB, Nuñez JA, Iglesias L, Ponce S. The new IASLC/ATS/ERS lung adenocarcinoma classification from a clinical perspective: current concepts and future prospects. J Thorac Dis 2014; 6: S526-36. doi: 10.3978/j.issn.20721439.2014.01.27.

13. Zhang M, Li X, Zhang X, Yang Y, Feng Z, Liu X. Association of serum hemoglobin A1c, C-peptide and insulin-like growth factor-1 levels with the occurrence and development of lung cancer. Mol Clin Oncol 2014; 2: 506-508.

14. Denko CW, Malemud CJ. Body mass index and blood glucose: correlations with serum insulin, growth hormone, and insulin -like growth factor-1 levels in patients with diffuse idiopathic skeletal hyperostosis (DISH). Rheumatol Int 2006; 26: 292-297. 